\title{
Experimental studies on the deformation and rupture of thin metal plates subject to underwater shock wave loading
}

\author{
Pengwan Chen a , Han Liu, Shaolong Zhang, Haibo Liu, Ang Chen, and Baoqiao Guo \\ State Key Laboratory of Explosion Science and Technology, Beijing Institute of Technology, Beijing 100081, China
}

\begin{abstract}
In this paper, the dynamic deformation and rupture of thin metal plates subject to underwater shock wave loading are studied by using high-speed 3D digital image correlation (3D-DIC). An equivalent device consist of a gas gun and a water anvil tube was used to supplying an exponentially decaying pressure in lieu of explosive detonation which acted on the panel specimen. The thin metal plate is clamped on the end of the shock tube by a flange. The deformation and rupture process of the metal plates subject to underwater shock waves are recorded by two high-speed cameras. The shape, displacement fields and strain fields of the metal plates under dynamic loading are obtained by using VIC-3D digital image correlation software. The strain gauges also were used to monitor the structural response on the selected position for comparison. The DIC data and the strain gauges results show a high level of correlation, and 3D-DIC is proven to be an effective method to measure 3D full-field dynamic response of structures under underwater impact loading. The effects of pre-notches on the failure modes of thin circular plate were also discussed.
\end{abstract}

\section{Introduction}

Plated structures are a kind of important basic element of marine structures and battleships, which may be subjected to an underwater explosion by the attack of a torpedo or a depth charge [1]. In ship design application, the ship plate shell with a small curvature is supported by welded stiffeners at its edges, so the shell between the stiffeners can be considered as a flat panel [2]. Non-contact underwater explosion is the important source of threat on the ship structure, and Understanding the response include deformation and failure modes subjected can supply an important reference for designing.

The response of clamped plate structures subjected to air and underwater blast loading has been studied for many years. In the underwater impact area, Ramajeyathilagam et al. [3-5] carried out many experimental and numerical investigations on the deformation and tensile tearing of airbacked metal plates and shell panels. The results indicated that the shock impulsive can be estimated in accordance with the Cole's empirical formula [6] and Taylor's plate theory [7]. Hung et al. [8] also presented experimental studies on the dynamic elastic response of the aluminium alloy panel subjected blast loading in the water tank. The research reports the underwater pressure history, the moving acceleration history and strain history of the plate surface.

For the underwater blast experimental study in laboratory scale, the reappear of underwater environment, production of the ideal blast impulsive loading and safety protection are all difficult and limited by the technology. With the development of technology, an experimental apparatus incorporating fluid-structure interaction (FSI) effects was recently developed to test scaled structures by

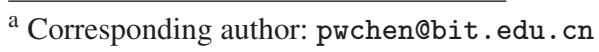

Espinosa et al. [9]. The set-up allows characterization of the response of solid and sandwich structures subjected to underwater blast impulsive loading. The calibration plate impact experiments confirmed that the FSI setup can generate an exponentially decaying pressure history. The full field out-of-plane deformation profile of annealed Steel plates was recorded in real time by Shadow Moiré and high speed photography. McShane et al. [10] also designed a similar equivalent device based on the impact produced the underwater shock wave loading. The potentiation of polymer coating for the resistence performance of the pure copper plates subjected underwater impact loading was analyzed by used this setup, and four typical failure models are recorded by highspeed photography.

However the level of understanding of the response of these structures at these high loading rates is not as established as that under static conditions. In this paper, A FSI apparatus was developed to generate an exponentially decaying pressure in lieu of explosive detonation by Xiang [11] in reference of Espinosa's works [9]. Combined with this equivalent device, 3D DIC technology and high-speed photography, the dynamic response of airbacked copper circular plates with pre-notches subjected to underwater impulsive loading was recorded in realtime. Compared with the strain gauge results and DIC results, the accuracy and advantage of DIC technology was proven obviously. The final failure modes for specimens with different pre-notches are evaluated by recycling the specimens. Moreover, the effects of pre-notches on the failure modes of thin circular plate were discussed. This work will be useful to those involved in research into the response of structures to explosive loading, a subject that has become increasingly important with heightened public awareness of potential explosive threats to civilian safety.

This is an Open Access article distributed under the terms of the Creative Commons Attribution License 4.0, which permits unrestricted use, distribution, and reproduction in any medium, provided the original work is properly cited. 


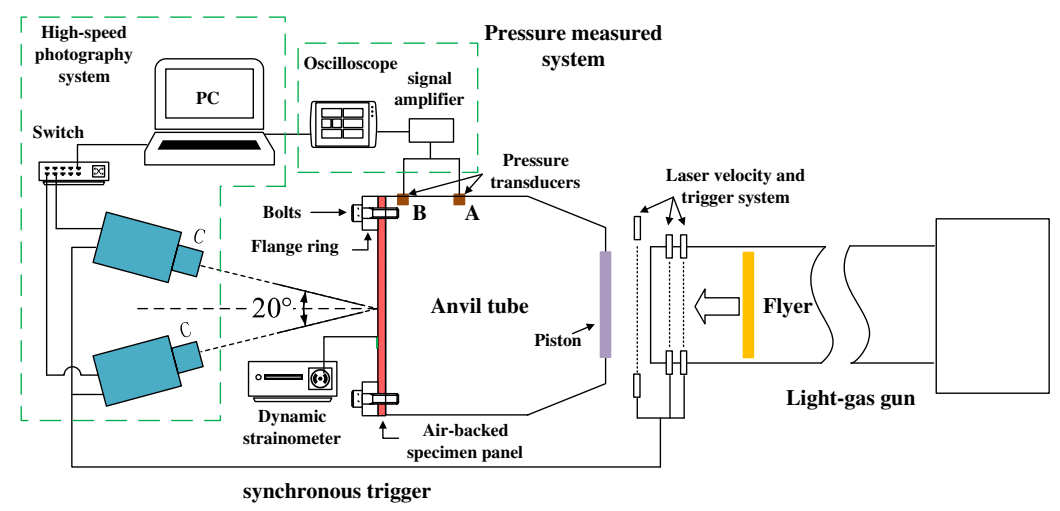

Figure 1. Principle diagram of the experiment setup.

(a)

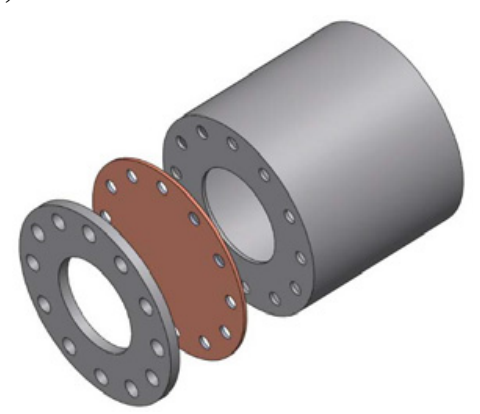

(b)

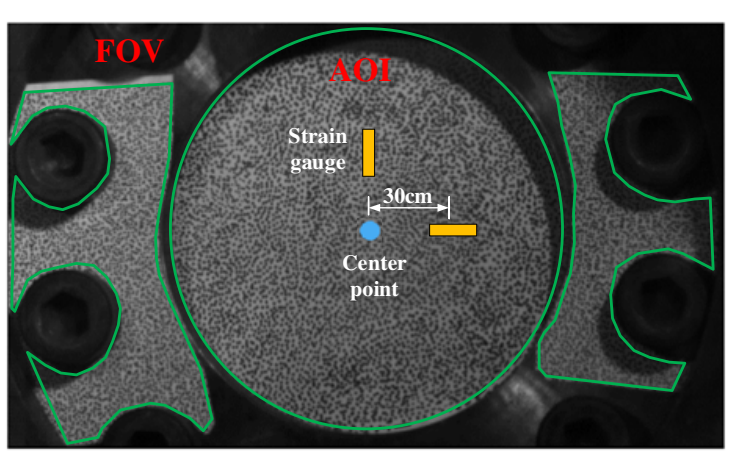

Figure 2. Principle diagram of the specimens: (a) the schematic of clamped support condition, (b) the schematic of selected computing area.

\section{Experimental details}

The simplified FSI equipment was developed by Xiang et al. (From School of Aerospace Engineering in Beijing Institute of Technology). The diagram of experimental setup is shown in Fig. 1. As seen from this figure, the simplified FSI equipment consists of two parts: a gas gun and a conical anvil tube filling up with water. A piston was sealing the conical anvil tube in the inlet of conical anvil tube opposite to the gas gun, and the disc specimens were clamped by the flange on the other bottom as shown in Fig. 2a. The flyer is driving by the gas gun and impact the piston, and the flyer speed and the piston thickness determine the peak pressure and decay time in the tube respectively [11]. In this paper, the strength of shock wave loading is control by changing the speed of the flyer (depended on the pressure of the gas gun). The speed of the flyer can be measured by two pairs of laser gauges position at the outlet of gas gun, and then the flyer fight through the laser trigger that synchronizer trigger the cameras. The pressure gauges respectively near the plate and in the center of conical anvil tube are used to measure the pressure of water shock wave during the loading event. So the peak pressure values respectively at a certain position A (in the anvil) can be predicted by Eq. (1), namely:

$$
\begin{aligned}
P_{A} & =\frac{Z_{w} Z_{p}}{Z_{w}+Z_{p}} V_{0}\left(\frac{D}{D_{A}}\right)^{2}, \\
Z_{w} & =40.82 \times 10^{6}, \quad Z_{p}=1.46 \times 10^{6} .
\end{aligned}
$$

Where $V_{0}$ is the the flyer impact velocity, $Z_{p}$ and $Z_{w}$ are the acoustic impedances of the piston of the piston and the water, where $D$ and $D_{A}$ are the diameters of the tube at the position A and impact locations.

Two Fastcam SA5 (produced by Photron Corporation in Japan) high-speed digital cameras were mounted in a stereo configuration (the angle is about $20^{\circ}$ ) to record the synchronized images of the deformation and rupture process of the sample specimens during the blast loading. The cameras are far from the explosive vessel and behind the observing windows in order to prevent vibration interference and protect the cameras during testing. In order to maximize the common field of view and the level of correlation, the two cameras were rotated and focused. The captured rate will be 50,000, and 75,000 frames per second. Three halogen lamps with a power of 1 kilowatt were used as the lighting source. The strain gauges were used to measurement the in-plane strain along radial and tangential directions shown in Fig. 2 b.

In this paper, two circular plates made of red copper with a diameter of $292 \mathrm{~mm}$ and same thickness of $1 \mathrm{~mm}$ were preparation before the testes subject to underwater shock wave loading. Through changing the types of the specimen surface (no pre-notch or pre-notch), the effect of pre-crack on the performance of specimens under underwater shock loading is understood with different pressure strength. The shapes of pre-crack is cross, and the position of pre-crack are in the center of plates. The length of the cross crack is $30 \mathrm{~mm}$, the width is $1 \mathrm{~mm}$, and the depth is $0.5 \mathrm{~mm}$. For comparison, a circular plate 
Table 1. Summary of experimental conditions of all specimens.

\begin{tabular}{|l|l|l|l|l|l|}
\hline No. & $\begin{array}{l}\text { Clamped } \\
\text { condition }\end{array}$ & $\begin{array}{l}\text { Pre- } \\
\text { notch }\end{array}$ & $V_{\mathbf{0}}(\mathbf{m} / \mathbf{s})$ & $P_{\mathbf{0}}(\mathbf{M P a})$ & $P_{\mathbf{B}}(\mathbf{M P a})$ \\
\hline I & fully & no & 152.8 & 83.5 & 91.1 \\
\hline II & fully & cross & 168.9 & 92.3 & 102.1 \\
\hline III & un-fully & cross & 101.9 & 55.7 & 61.2 \\
\hline
\end{tabular}
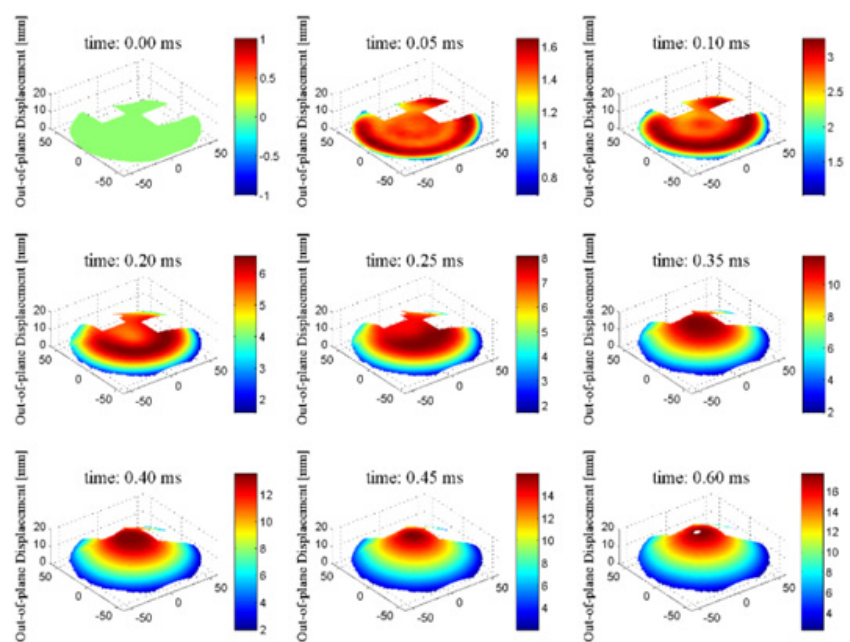

Figure 3. Principle diagram of the experiment setup.

with a diameter of $200 \mathrm{~mm}$ and same size cross prenotch subjected a lower loading are also tested, and the specmen was only fixed while bolts not through the plate, hereinafter referred to as "un-fully clamped". Table 1 is a summary of experimental conditions of all specimens, including the support condition, the pre-notch of the metal plates, the impact velocity $V_{0}$, the theoretical peak pressure $P_{0}$ and the measured peak pressure at position A $P_{A}$.

Before prepared the random speckle of samples, the surface must be cleaned and polished. Then, the high contrast speckle pattern is prepared on the plate by spraying white paint and marking the random points by a black marker pen on the plate surface, and the black speckle dots with a diameter of about 5 pixels when seen by two cameras are thought perfect for the random highcontrast pattern. As shown in Fig. 2b, three areas on the specimen surface and flange surface were selected as the AOI (area of interest) which is used to analyze the deformation fields by comparing the difference before and after deformation. During the shock, the shock absorbers cannot completely prevented the motion of the anvil tube. So the DIC measured motion on the specimen surface is absolute displacement include the plate deflection and the tube rigid motion. The areas on the flange surface were used to calculate the rigid displacement of the anvil tube.

\section{Results and discussion}

\subsection{DIC results of no pre-notched plate}

The evolution of displacement $(W$ ) fields on the surface of no pre-notched specimen at different moments are

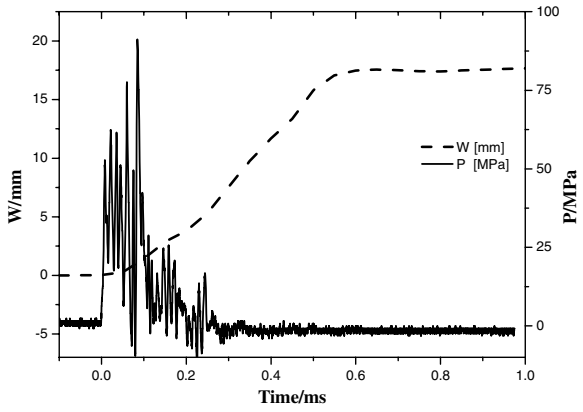

Figure 4. Diagram of the deflection history of point $\mathrm{O}$ and the pressure history of position B.

shown in Fig. 3. The major component is the out-ofplane displacement (absolute displacement). At $0.05 \mathrm{~ms}$, a visible symmetric deformation ring can be found in the boundary of AOI, and then the deformation region rapidly spreads to the center along the radial direction until $0.25 \mathrm{~ms}$. It is worth noticed that the shape of the deformation area is flat nearly except the deformation ring in the first, and evolves into a symmetric curved shape after $0.35 \mathrm{~ms}$ with the propagation of deformation ring appeared in the boundary. The displacement ring can be seen as plastic yield line of the first bending modal mode shape of the plate.

Figure 4 shows the comparison between the deflection history of centeral point $\mathrm{O}$ and the pressure history of position B for the same specimen. The initial moment of pressure history of position $\mathrm{B}$ is unified with the initial moment of the pressure history loading on the wet side of the specimen. At $0.1 \mathrm{~ms}$, the first loading from the incident shock wave has vanished, the plate continuous to deform due to multiple followed incident and reflected shock waves and the inertial effects of the plates. It is indicated that the resultant plastic deformation is caused by the combined effect of the reflected shock, the incident shock and the support condition of the structure.

Strain is used to describe the relative change of the material's shape as a normalization of the deformation [35]. The evolution of the in-plane principal strain fields of the no pre-notched specimen at different moments is shown in Fig. 5. It can be seen that until $0.15 \mathrm{~ms}$ the higher principal strains occur close to the boundaries. During the loading, the incident underwater impulse forces all points of the panel to move out. But points close the clamping area are constrained and higher strains occur in the boundary areas. Further, when inertia forces and the reflection waves are taking over, strains are developing in the centre of the plate reaching first peak values 5 at $0.55 \mathrm{~ms}$ in Fig. 5. Somewhat counter-intuitively, the entire central and boundary region of the field of view is at a higher strain than the surrounding plastically deformed circular region in follow.

Figure 6 shows the comparison of the normal strains $\varepsilon_{x x}$ and $\varepsilon_{y y}$ between the DIC results and the strain gauge results on the strain gauge locations as shown in Fig. 2. The DIC results are acquired from the strain date of symmetric points to the center. From this figure, the strain histories of $\varepsilon_{x x}$ and $\varepsilon_{y y}$ all correlate well until $0.6 \mathrm{~ms}$ between the DIC and the strain gauge. After this moment, the 


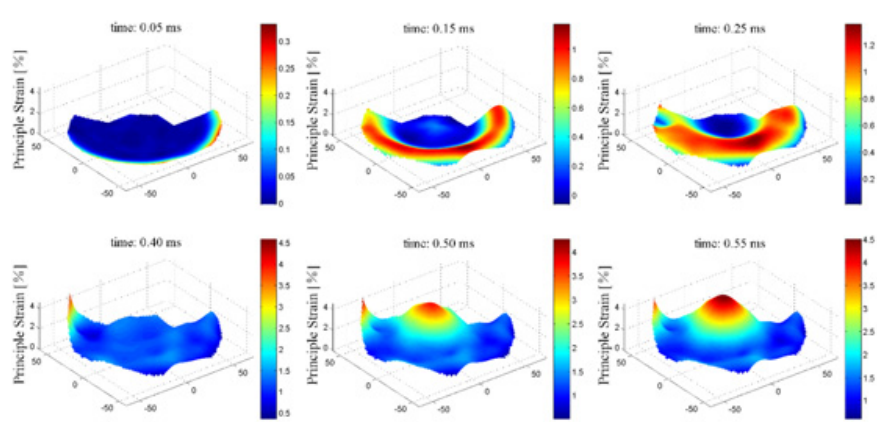

Figure 5. Full-field resultant in-plane principal strain at different time steps.

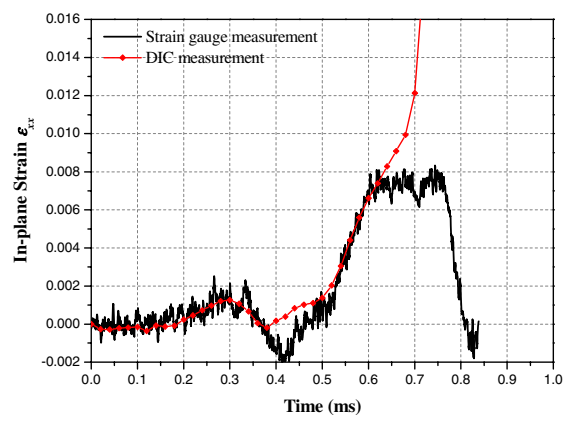

(a) histories of $\varepsilon_{x x}$

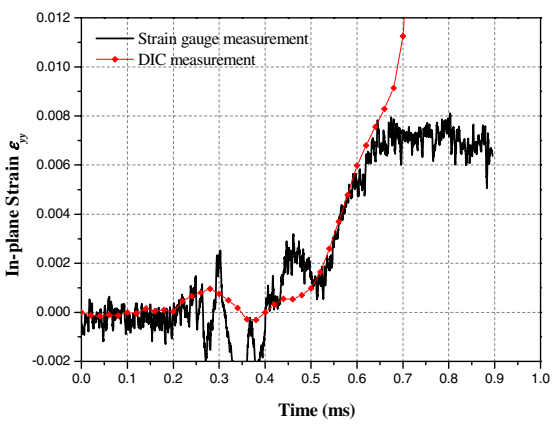

(b) histories of $\varepsilon_{y y}$

Figure 6. Comparison of the normal strains $\varepsilon_{x x}$ and $\varepsilon_{y y}$ between the DIC results and the strain gauge results.

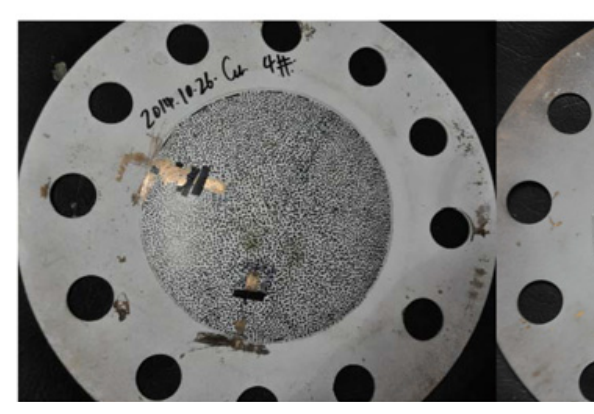

(a) specimen I

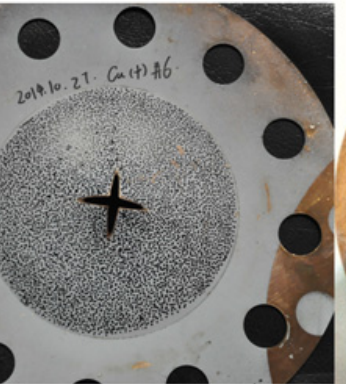

b) specimen II

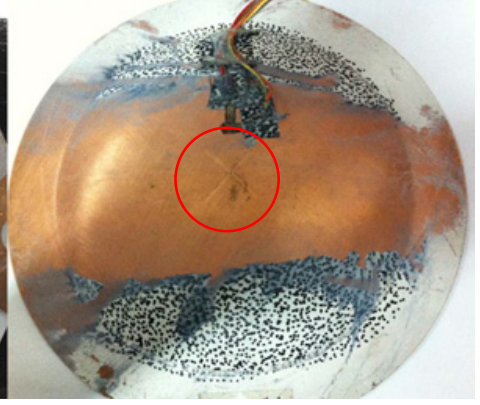

(c) specimen III

Figure 7. Failure modes observed for different specimens.

strain history curves stop increasing and even decrease, possibility caused by the strain gauges falling off from the specimen surface. The comparison indicates that DIC technology is a dependable measured method.

\subsection{Recovering and discussion}

After the blast tests, all specimens were collected for further analysis. Figure 7 shows the photographs of four recovered specimens after tests. Three types of failure modes can be identified. For the specimen without prenotch, only large ductile deformation can be observed in Fig. 7a; for the pre-notched specimen, large ductile deformation and local petalling at the location of prenotch can be observed in Fig. 7b; for the un-fully clamped specimen, in addition to large ductile deformation, local necking in the center can also be observed in Fig. 7c, which is confined to the location of the pre-notch.

\section{Conclusions}

This paper focus on the dynamic deformation and rupture of pre-notched circular copper plates subjected to underwater impulse loading. High-speed photography, combined with 3D-DIC has been successfully used to acquire the out-of-plane displacement and in-plane principal strain on the surface of the circular plates during the blast process. Compared with the strain gauge results and DIC results, the accuracy and advantage of DIC technology was proven obviously. Through change the clamped condition and the loaded intensity, the evolution of the failure modes has been observed and discussed. Moreover, the effects of pre-notches on the failure modes of thin circular plate were discussed. 3D-DIC is also proven to be an effective method to measure 3D full-field dynamic response of structures under underwater impact loading. 


\section{References}

[1] Rajendran, R.L.J. Marine Structures, 22, 2 (2009)

[2] EN, F.. ONR, 1 (1947)

[3] Ramajeyathilagam K, V.C.P., International Journal of Impact Engineering (2004)

[4] Ramajeyathilagam, K., P.V. C and B.R. V.. International Journal of Impact Engineering, 24, 10 (2000)

[5] Ramajeyathilagam, K., C.P.. Shock and Vibration, 8, 5 (2001)

[6] RH, C., Underwater explosions (Dover Publications Inc, New York, USA, 1948)
[7] GI, T.. Compendium Underwater Explosion Res ONR, 1: p. 1155-74 (1950)

[8] Hung, C.F., P.Y. Hsu and J.J. Hwang-Fuu. International Journal of Impact Engineering, 31, 2 (2005)

[9] Espinosa, H.D., S. Lee and N. Moldovan, Experimental Mechanics, 46, 6 (2006)

[10] McShane, G.J., et al., International Journal of Solids and Structures, 45, 16 (2008)

[11] Xiang Da-lin, Rong Ji-li, He Xuan, Hu Chang-hua, Li Jian, Zhang Wei, Ren Peng, Acta Armamentarii (in CHINA), 39, 6 (2014) 